\title{
Theory of electronic transport through a triple quantum dot in the presence of magnetic field
}

\author{
F. Delgado \\ Quantum Theory Group, Institute for Microstructural Sciences, \\ National Research Council, Ottawa, Ontario, Canada K1A OR6 and \\ Department of Physics, University of Ottawa, \\ MacDonald Hall, 150 Louis Pasteur, \\ Ottawa, Ontario, Canada K1N 6N5 \\ P. Hawrylak \\ Quantum Theory Group, Institute for Microstructural Sciences, \\ National Research Council, Ottawa, Ontario, Canada K1A OR6
}

\begin{abstract}
Theory of electronic transport through a triangular triple quantum dot subject to a perpendicular magnetic field is developed using a tight binding model. We show that magnetic field allows to engineer degeneracies in the triple quantum dot energy spectrum. The degeneracies lead to zero electronic transmission and sharp dips in the current whenever a pair of degenerate states lies between the chemical potential of the two leads. These dips can occur with a periodicity of one flux quantum if only two levels contribute to the current or with half flux quantum if the three levels of the triple dot contribute. The effect of strong bias voltage and different lead-to-dot connections on Aharonov-Bohm oscillations in the conductance is also discussed.
\end{abstract}

PACS numbers: 73.21.La,73.23.Hk 


\section{INTRODUCTION}

Using charge sensing techniques Gaudreau et al. $\stackrel{12}{2}^{2}$ recently demonstrated lateral triple quantum dot (TQD) molecule with controlled number of electrons, down to zero. Preliminary transport experiments in external magnetic field ${ }^{3,4}$ showed signatures of AharonovBohm (AB) oscillations, indicating coherent coupling between the constituent dots. Motivated by forthcoming experiments, we present here a theory describing signatures of $\mathrm{AB}$ oscillations in transport through the TQD in a perpendicular magnetic field. Using tight binding model we show that magnetic field allows us to engineer degeneracies in the triple quantum dot spectrum, and that these degeneracies lead to zero electronic transmission and to sharp dips in the current. These anomalies in transport can appear with different periodicities or be suppressed depending on the applied source-drain voltage and dot energies. The main features of the transport are explained as an interplay between Fano resonances and $\mathrm{AB}$ oscillations. The $\mathrm{AB}$ oscillations apparent in the conductance allows for unambiguous identification of TQD parameters. The effects of strong bias voltage on the conductance are also discussed. Two different lead-to-dot connections are considered: a left lead connected to a single dot and left lead connected to two dots. The first configuration leads to a periodic oscillation of the current with the magnetic field while the second one breaks the periodicity introducing an extra structure superimposed on the oscillatory behaviour as a function of the magnetic flux.

In our tight-binding model, effects associated with the electron-electron interactions, extensively analyzed in the context of transport through single quantum dots, experimentally $\underline{\underline{5}, 6,7}$ and theoretically, $, \underline{\underline{8}}, \underline{10,11,12,13}$ in relation with the Kondo physics do not appear. However, broadening of molecular energy levels is properly taken into account in our model. A perpendicular magnetic field is accounted for by Peierls phase factors $\frac{14,15}{12}$ in the single-particle tunneling elements, leading to AB oscillations in the conductance with period of one flux quantum $\Phi_{0}=e / h c$ ( $e$ - electron charge, $h$-Plank's constant and $c$ - speed of light), and anomalies at half flux quantum. The AB oscillations in the conductance are in-

herent to rings threaded by magnetic flux. $\stackrel{16,17}{1}$ Flux period of $\Phi_{0}$ is observed in conventional AB experiments with electrons propagating in field-free regions 18,19 and also in mesoscopic experiments, for example in metal rings ${ }^{20}$ or in electronic Mach-Zehnder interferometers. $\underline{21}^{22}$ Furthermore, $\Phi_{0} / 2$ periods can be also observed due to weak localization effects $\underline{23}, 24$ 
At difference with previous works on equilateral triple dot connected to leads where only the linear response to a small bias was analyzed, $25,26,27$ or works based on a master equation approach to a single electron tunneling $28,29,30$ valid only in the limit of large applied bias, we discuss the differential conductance in the case of arbitrary applied bias voltage and magnetic field in an exact non-perturbative way, including the experimental conditions in Ref. 3 .

The paper is organized as follows. In Sec. II we introduce the Hamiltonian describing the system while Sec. III explains how to obtain the transmission coefficient from the transfer matrix and the scattering boundary conditions. The AB oscillations in the current are analyzed in Sec. IIIA together with the Fano line-shape of the transmission probability while the anomalous behaviour of the transmission close to multiples of half flux quantum is studied in Sec. IIIB. The conductance in the non-linear regime is analyzed in Sec. V] The paper is summarized in Sec. VI,

\section{MODEL}

The triple dot connected to leads is plotted schematically in Fig. 11. The leads are described within a one-dimensional tight-binding model, with nearest neighbors hopping $t_{L}$. Each dot is represented by a single orbital, connected to nearest neighbors by magnetic field dependent hopping matrix elements $t_{i j}(B)$, with $i, j=1,2,3(i \neq j)$. The left lead is connected to the dots 1 and 2, see Fig. 1, through the hopping elements $t_{L 1}$ and $t_{L 2}$, while the right lead is connected only to dot 3 with hopping matrix element $t_{R 3}$. The TQD is subject to a uniform perpendicular magnetic field $\mathbf{B}, \mathbf{B}=B \hat{\mathbf{z}}$. The Hamiltonian describing the system is then given by

$$
H=H_{T Q D}+H_{l e a d s}+H_{L D}
$$

where $H_{T Q D}$ is the Hamiltonian corresponding to an electron in an isolated triple dot

$$
H_{T Q D}=\sum_{i=1}^{3}(E-\Delta V / 2) d_{i}^{+} d_{i}+\sum_{i, j=1, i \neq j}^{3} t_{i j}(B) d_{i}^{+} d_{j}
$$

the operators $d_{i}\left(d_{i}^{+}\right)$annihilate (create) an electron in dot $i$. $E$ is the energy level of each quantum dot and $\Delta V$ is the energy bias between the two leads. Notice that as a first order 
approximation, we have assumed that the shift in the dot energy levels as a function of the applied bias is the same for all dots, $-\Delta V / 2$. Furthermore, for identical dots the hopping matrix elements at $B=0$ satisfy $t_{i j}=t \forall i, j$.

$H_{\text {leads }}$ is the Hamiltonian describing the two non-interacting leads with $N$ sites each,

$$
\begin{aligned}
H_{\text {leads }} & =\epsilon_{L} c_{0}^{+} c_{0}+\sum_{i=-N+1}^{-1}\left[\epsilon_{L} c_{i}^{+} c_{i}+t_{L}\left(c_{i}^{+} c_{i+1}+c_{i+1}^{+} c_{i}\right)\right] \\
& +\epsilon_{R} c_{1}^{+} c_{1}+\sum_{i=2}^{N}\left[\epsilon_{R} c_{i}^{+} c_{i}+t_{L}\left(c_{i-1}^{+} c_{i}+c_{i}^{+} c_{i-1}\right)\right],
\end{aligned}
$$

where $c_{i}^{+}$and $c_{i}$ are respectively the creation and annihilation operators of an electron on site $i$ in the leads, $\epsilon_{L}$ is the on-site energy in the leads at zero bias and $\epsilon_{R}=\epsilon_{L}-\Delta V$. Both leads are characterized by the same hopping matrix elements, $t_{L}$. Finally, the interaction Hamiltonian $H_{L D}$ is given by

$$
H_{L D}=t_{L 1}(B) c_{0}^{+} d_{1}+t_{L 2}(B) c_{0}^{+} d_{2}+t_{R 3}(B) c_{1}^{+} d_{3}+h c .
$$

The magnetic field $B$ renormalizes the single-particle tunneling elements $t_{j k}$ by Peierls phase factors, $\underline{\underline{14}, \underline{15}} t_{j k}(B)=t_{j k} e^{2 \pi i \phi_{j k}}$, where $\phi_{j k}=\frac{e}{2 \pi \hbar c} \int_{\mathbf{R}_{j}}^{\mathbf{R}_{k}} \mathbf{A} . d \mathbf{l}$. $\mathbf{A}$ is the corresponding vector potential and $\mathbf{R}_{j}$ and $\mathbf{R}_{k}$ are the positions of the sites connected by the hopping elements $t_{j k}$ Taking the symmetric gauge in which $\mathbf{A}=[-B y, B x, 0]$, the phase difference between two points $\mathbf{R}_{j}$ and $\mathbf{R}_{k}$ is given by $\phi_{j k}=\frac{1}{2} \mathbf{B} \cdot\left(\mathbf{R}_{k} \times \mathbf{R}_{j}\right) \Phi_{0}$, see Fig. 2, with $\Phi_{0}=\frac{h c}{e}$ the magnetic flux quantum.

For the three quantum dots located in the corners of an equilateral triangle we have $\phi_{12}=\phi_{23}=\phi_{31}=-\phi / 3$. Here, $\phi=3 \sqrt{3} B R^{2} / 4 \Phi_{0}$ is the number of magnetic flux quanta threading the area of the TQD, with $R$-the distance from the center of the triangle to each dot, identified in Fig. 1.

For the general case where the left lead is connected to dots one and two through the hopping matrix elements $t_{L 1}(B)$ and $t_{L 2}(B)$ respectively, while dot three is connected only to the right lead with hopping parameter $t_{R 3}$, as shown in Fig. 1, there is an extra magnetic flux $\phi^{\prime}$. In this case, $t_{L 1}(B)=t_{L 1} e^{2 \pi i \phi^{\prime}}$ and $t_{L 2}(B)=t_{L 2} e^{-2 \pi i \phi^{\prime}}$. If $S_{1}$ and $S_{2}$ are the shaded areas on Fig. 1, the two fluxes are related through the ratio of areas $\phi^{\prime}=-\phi \frac{4 \pi^{2}}{3 \sqrt{3}}\left(\frac{S_{1}+S_{2}}{\pi R^{2}}\right)$. As it will be shown, these phases have an important effect on transport leading to a nonperiodic behaviour of the transmission with the magnetic field, except for the particular case where $\phi / \phi^{\prime}$ is a rational number. 


\section{TRANSFER MATRIX AND SCATTERING MATRIX}

Our aim in the present section is to obtain a $2 \times 2$ transfer matrix $\mathcal{T}$ which relates the amplitude of the wave functions on the last two sites of the left lead, $C_{-1}$ and $C_{0}$, with those at the first two positions of the right lead, $C_{1}$ and $C_{2}$. In matrix form,

$$
\left(\begin{array}{cc}
\mathcal{T}_{11} & \mathcal{T}_{12} \\
\mathcal{T}_{21} & \mathcal{T}_{22}
\end{array}\right) \cdot\left(\begin{array}{c}
C_{-1} \\
C_{0}
\end{array}\right)=\left(\begin{array}{c}
C_{1} \\
C_{2}
\end{array}\right)
$$

Here and in the following sections, we will use the notation $X_{i} \equiv\left\langle\mathbf{r}\left|x_{i}^{+}\right| 0\right\rangle$ with $x_{i}^{+}=c_{i}^{+}, d_{i}^{+}$ for the amplitude of the wave function at position $i$. The transmission and reflection coefficients can be then obtained by imposing the scattering boundary conditions. In particular, if we consider a left incident plane wave with wavevector $k$, the amplitudes at the left and right of the triple dot will be given by

$$
\begin{aligned}
C_{0} & =1+R \\
C_{-1} & =e^{-i k a}+R e^{i k a} \\
C_{1} & =T \\
C_{2} & =T e^{i k^{\prime} a},
\end{aligned}
$$

The wavevector and the energy of the incident electron $\varepsilon$ is related through the dispersion

relation in an infinite lead, $k a=\operatorname{arc} \cos \left(\frac{\varepsilon-\epsilon_{L}}{2 t_{L}}\right)$ and $k^{\prime} a=\operatorname{arc} \cos \left(\frac{\varepsilon-\epsilon_{L}-\Delta V}{2 t_{L}}\right)$, with $a$ the lattice constant. Then, from Eqs. (5) and (6) , reflection $R$ and transmission $T$ can be expressed as:

$$
\begin{aligned}
& R=\frac{e^{-i k a}\left[-e^{i k^{\prime} a} \mathcal{T}_{11}+\mathcal{T}_{21}+e^{i k a}\left(-e^{i k^{\prime} a} \mathcal{T}_{12}+\mathcal{T}_{22}\right)\right]}{e^{i k^{\prime} a}\left(e^{i k a} \mathcal{T}_{11}+\mathcal{T}_{12}\right)-\left(e^{i k a} \mathcal{T}_{21}+\mathcal{T}_{22}\right)} \\
& T=\frac{e^{-i k a}\left(-1+e^{2 i k a}\right)\left(-\mathcal{T}_{12} \mathcal{T}_{21}+\mathcal{T}_{11} \mathcal{T}_{22}\right)}{e^{i k^{\prime} a}\left(e^{i k a} \mathcal{T}_{11}+\mathcal{T}_{12}\right)-\left(e^{i k a} \mathcal{T}_{21}+\mathcal{T}_{22}\right)}
\end{aligned}
$$

The transfer matrix $\mathcal{T}$ will be obtained by applying the Hamiltonian to the amplitudes. It is convenient to express the original Hamiltonian (1) in the basis of eigenfunctions of the isolated triple dot. If we define the annihilation operators $\bar{d}_{1}, \bar{d}_{2}, \bar{d}_{3}$ in terms of the corresponding annihilation operators for electrons on sites 1,2 and 3 as

$$
\left\{\begin{array}{l}
d_{1}=\frac{1}{\sqrt{3}}\left(\bar{d}_{1}+\bar{d}_{2}+\bar{d}_{3}\right) \\
d_{2}=\frac{1}{\sqrt{3}}\left(\bar{d}_{1}+e^{-2 \pi i / 3} \bar{d}_{2}+e^{2 \pi i / 3} \bar{d}_{3}\right) \\
d_{3}=\frac{1}{\sqrt{3}}\left(\bar{d}_{1}+e^{2 \pi i / 3} \bar{d}_{2}+e^{-2 \pi i / 3} \bar{d}_{3}\right)
\end{array}\right.
$$


the triple dot Hamiltonian will be diagonal at all values of the magnetic field:

$$
\bar{H}_{T Q D}=\epsilon_{1} \bar{d}_{1}^{+} \bar{d}_{1}+\epsilon_{2} \bar{d}_{2}^{+} \bar{d}_{2}+\epsilon_{3} \bar{d}_{3}^{+} \bar{d}_{3}
$$

where $\epsilon_{1}=\left[E-\frac{\Delta V}{2}-2|t| \cos \left(\frac{2 \pi \phi}{3}\right)\right], \quad \epsilon_{2}=\left[E-\frac{\Delta V}{2}-2|t| \cos \left(\frac{2 \pi(\phi+1)}{3}\right)\right]$ and $\epsilon_{3}=$ $\left[E-\frac{\Delta V}{2}-2|t| \cos \left(\frac{2 \pi(\phi-1)}{3}\right)\right]$. Notice that the eigenvalues of the triple dot Hamiltonian depend on the magnetic field. For the TQD-leads coupling Hamiltonian, $H_{L D}$, we obtain

$$
\begin{aligned}
\bar{H}_{L D}= & \bar{t}_{L 1} c_{0}^{+} \bar{d}_{1}+\bar{t}_{L 2} c_{0}^{+} \bar{d}_{2}+\bar{t}_{L 3} c_{0}^{+} \bar{d}_{3} \\
& +\bar{t}_{R 1} c_{1}^{+} \bar{d}_{1}+\bar{t}_{R 2} c_{1}^{+} \bar{d}_{1}+\bar{t}_{R 3} c_{1}^{+} \bar{d}_{3}+h c .
\end{aligned}
$$

The new tunneling elements $\bar{t}_{L j}$ and $\bar{t}_{R j}$ are given by

$$
\begin{aligned}
\bar{t}_{L 1} & =\frac{1}{\sqrt{3}}\left(t_{L 1}+t_{L 2}\right) \\
\bar{t}_{L 2} & =\frac{1}{\sqrt{3}}\left(t_{L 1}+e^{-2 \pi i / 3} t_{L 2}\right)=\bar{t}_{L 3}^{*} \\
\bar{t}_{R 1} & =\frac{t_{R 3}}{\sqrt{3}} \\
\bar{t}_{R 2} & =\frac{t_{R 3}}{\sqrt{3}} e^{2 \pi i / 3}=\bar{t}_{R 3}^{*} .
\end{aligned}
$$

In Eqs. (10) and (11) we have omitted the magnetic flux dependence of the tunneling matrix elements between the dots and the leads in order to simplify the notation. In fact, this dependence does not appear when the left lead is connected only to one dot, case that we shall analyze with more detail later.

Defining the amplitudes $\bar{D}_{i}=\left\langle\mathbf{r}\left|\bar{d}_{i}^{+}\right| 0\right\rangle$, the Schrödinger equation reads as

$$
\left\{\begin{array}{l}
t_{L} C_{-1}+\left(\epsilon_{L}-\varepsilon\right) C_{0}+\bar{t}_{L 1} \bar{D}_{1}+\bar{t}_{L 2} \bar{D}_{2}+\bar{t}_{L 3} \bar{D}_{3}=0 \\
\bar{t}_{L 1}^{*} C_{0}+\left(\epsilon_{1}-\varepsilon\right) \bar{D}_{1}+\bar{t}_{R 1}^{*} C_{1}=0 \\
\bar{t}_{L 2}^{*} C_{0}+\left(\epsilon_{2}-\varepsilon\right) \bar{D}_{2}+\bar{t}_{R 2}^{*} C_{1}=0 \\
\bar{t}_{L 3}^{*} C_{0}+\left(\epsilon_{3}-\varepsilon\right) \bar{D}_{3}+\bar{t}_{R 3}^{*} C_{1}=0 \\
\bar{t}_{R 1} \bar{D}_{1}+\bar{t}_{R 2} \bar{D}_{2}+\bar{t}_{R 3} \bar{D}_{3}+\left(\epsilon_{L}-\Delta V-\varepsilon\right) C_{1}+t_{L} C_{2}=0
\end{array} .\right.
$$

Eq. (12) allows us to express the amplitudes $C_{1}, C_{2}$ as a function of $C_{-1}$ and $C_{0}$. In so doing, one has to substitute the expressions for $\bar{D}_{i}$ in terms of the amplitudes in the leads and write the resulting relations as in Eq. (5). To simplify the expressions, in all the following discussions we will fix $\epsilon_{L}=0$ and the energy scale such as $t_{L}=-1$, which implies 
that the energy band of the leads is from -2 to 2 . Then,

$$
\begin{aligned}
& \mathcal{T}_{11}=\left[\frac{\bar{t}_{L 1} \bar{t}_{R 1}^{*}}{\varepsilon-\epsilon_{1}}+\frac{\bar{t}_{L 2} \bar{t}_{R 2}^{*}}{\varepsilon-\epsilon_{2}}+\frac{\bar{t}_{L 3} \bar{t}_{R 3}^{*}}{\varepsilon-\epsilon_{3}}\right]^{-1} \\
& \mathcal{T}_{12}=-\mathcal{T}_{11}\left[-\varepsilon+\frac{\left|\bar{t}_{L 1}\right|^{2}}{\varepsilon-\epsilon_{1}}+\frac{\left|\bar{t}_{L 2}\right|^{2}}{\varepsilon-\epsilon_{2}}+\frac{\left|\bar{t}_{L 3}\right|^{2}}{\varepsilon-\epsilon_{3}}\right] \\
& \mathcal{T}_{21}=\mathcal{T}_{11}\left[-\varepsilon+\frac{\left|\bar{t}_{R 1}\right|^{2}}{\varepsilon-\epsilon_{1}}+\frac{\left|\bar{t}_{R 2}\right|^{2}}{\varepsilon-\epsilon_{2}}+\frac{\left|\bar{t}_{R 3}\right|^{2}}{\varepsilon-\epsilon_{3}}\right] \\
& \mathcal{T}_{22}=\frac{1}{\mathcal{T}_{11}^{*}}-\frac{\mathcal{T}_{12} \mathcal{T}_{21}}{\mathcal{T}_{11}}
\end{aligned}
$$

Equation (13) is the central result of the paper. Nevertheless, the expressions for the transmission and reflection coefficients using Eqs. (13) and (7) are still too lengthy, so we shall analyze several particular cases.

In the following subsections, we study two particular cases that can be handled analytically. To get a clear understanding of the main features of the transmission we will consider the simplest case where $t_{L 2}=0$ and $t_{L 1}=t_{R 3}=t_{L D}$. We shall further simplify the problem assuming zero bias voltage.

\section{A. Transmission on-resonance with a single level}

Let us consider first the situation where the incident energy is very close to one of the levels, e.g., level 1. Furthermore, we will assume that the other two levels are far way, i.e., $\left|t_{L D}\right| \sim\left|\epsilon_{1}-\varepsilon\right|<<\left|\epsilon_{2}-\varepsilon\right|,\left|\epsilon_{3}-\varepsilon\right|$. Under these conditions, the Hamiltonian (1)

reduces to the Fano-Anderson mode ${ }^{31}$ of a localized state in the continuum. In this case, the tranmission close to level 1 is given by a Fano like resonance ${ }^{31}$

$$
|T(\varepsilon)|^{2} \propto \frac{\left(q \Gamma / 2+\varepsilon-\epsilon_{1}\right)^{2}}{\left(\varepsilon-\epsilon_{1}\right)^{2}+\Gamma^{2} / 4},
$$

where $q$ is the Fano parameter and $\Gamma$ the width of the resonance defined in Ref. 32. If the three levels are far apart, each level will lead to one of this Fano-resonances with their respective central energy, Fano parameter and widths. Although we have used an implicit notation in which the magnetic flux dependence is not apparent, we should emphasize that the main variation of the transmission with the magnetic flux in this single-resonance regime is governed by the sinusoidal variation of the single particle energy levels $\epsilon_{i}$, Eq. (9), with $\phi$. 
Fig. 3 shows the logarithm of the transmission probability versus the incident energy at zero magnetic field for the single lead-to-dot connection (solid line) and the double connection (dashed line), as well as the corresponding fitting to the form of Eq. (14) (dots and diamonds, respectively). For this case, where the coupling between the leads and the dots is quite small compared to the tunneling $t_{L}$, the line shape is quasi-Lorentzian, as indicating the high values of $q$.

\section{B. Transmission close to a degenerate level}

Our aim now is to study the effects of the magnetic field induced degeneracies of the triple dot on the transmission. When the energy of the incident electrons is close to the quasi degenerate level, the effect of the third orbital of the triple dot on the transmission can be neglected, see lower panel of Fig. 4. This approximation is valid for incident energies

such $\tilde{E}-2\left|t_{L D}\right| \leq \varepsilon \leq \tilde{E}+2\left|t_{L D}\right|$, where $\tilde{E}$ is the energy level of the degenerate states. The elements of the transfer matrix can be obtained from Eq. (13) and, after the substitution in Eq. (7) and some extra algebra, the transmission probability reads as

$$
\begin{aligned}
& |T(\delta)|^{2}= \\
& \frac{\left(-1+e^{2 i k a}\right)^{2} t_{L D}^{4} \delta^{2}}{\beta\left[t_{L D}^{2}-3 \delta\left(e^{i k a}+\tilde{E}+\delta\right)\right]\left[t_{L D}^{2}-\delta\left(e^{i k a}+\tilde{E}+\delta\right)\right] \alpha(\delta)\left[\alpha(\delta)+2 \delta+2 e^{i k a} \delta(\tilde{E}+\delta)\right]},
\end{aligned}
$$

where $\beta=\left(-1+e^{i \pi / 3}\right)\left(1+e^{2 i \pi / 3}\right)$ and $\alpha(\delta)=\delta+e^{i k a}\left[-t_{L D}^{2}+\delta(\tilde{E}+\delta)\right]$. Here we have defined the energy shift $\delta=\varepsilon-\tilde{E}$ and the corresponding wavevector $k(\delta) a=\arccos [(\delta+$ $\tilde{E}) / 2$. Notice that we have written the previous expression in an apparently complex form, but it can be checked that Eq. (15) provides a real positively defined quantity. Although this expression is still quite complicated, it is clear that the transmission probability goes to zero when we are on-resonance $\left(|T|^{2} \propto \delta^{2}\right)$. This result was previously described in the context of scattering through a tunneling junction with two resonant impurities in Ref. 33 . In fact, when the tunneling $t_{L D}$ is small enough, i.e. $\left|t_{L D}\right|<<1$, and under the assumption $|t|<<1$, the transmission probability when the degenerate orbital level is on-resonance with 
the Fermi energy of the leads $(\tilde{E}=0)$, can be expressed as

$$
|T(\delta)|^{2} \approx \frac{2 \delta^{2} \Gamma(\delta) \frac{t_{L D}^{4}}{\left(t_{L D}^{4}+\delta^{2}\right)\left(t_{L D}^{4}+9 \delta^{2}\right)}}{\left(-\delta^{2}+\frac{\Gamma(\delta)^{2}}{4}\right)},
$$

where

$$
\Gamma(\delta)=\frac{2\left[t_{L D}^{4}-2\left(-5+2 t_{L D}^{2}\right) \delta^{2}+\frac{9 \delta^{4}}{t_{L D}^{4}}\right] t_{L D}^{4}}{\left(t_{L D}^{4}+\delta^{2}\right)\left(t_{L D}^{4}+9 \delta^{2}\right)} .
$$

As we can see from Eq. (16), the transmission probability close to the degenerate level $\tilde{E}$ can not be approximated by the addition of two Fano resonances, as one would naively expect from Eq. (14).

Let us analyze why the transmission coefficient goes to zero when the incident particles are on-resonance with a degenerate level. Let us consider arbitrary (but small) tunneling elements $\bar{t}_{L j}, \bar{t}_{R j}$ such that the two level approximation is still valid. Without loss of generality, we assume that the degenerate levels are $\epsilon_{1}$ and $\epsilon_{2}$. Then, the Schrödinger equation for the incident energy $\varepsilon=\tilde{E}=0$ can be written as

$$
\left\{\begin{array}{l}
-C_{-1}+\bar{t}_{L 1} \bar{D}_{1}+\bar{t}_{L 2} \bar{D}_{2}=0 \\
\bar{t}_{L 1}^{*} C_{0}+\bar{t}_{R 1}^{*} C_{1}=0 \\
\bar{t}_{L 2}^{*} C_{0}+\bar{t}_{R 2}^{*} C_{1}=0 \\
-C_{2}+\bar{t}_{R 1} \bar{D}_{1}+\bar{t}_{R 2} \bar{D}_{2}=0
\end{array}\right.
$$

The system of equations (17) admits two kinds of solutions depending on the value of the determinant

$$
A=\operatorname{det}\left(\begin{array}{ll}
\bar{t}_{L 1}^{*} & \bar{t}_{R 1}^{*} \\
\bar{t}_{L 2}^{*} & \bar{t}_{R 2}^{*}
\end{array}\right)
$$

Let us consider first the case where $A=0$. This implies that $\bar{t}_{L 1} \bar{t}_{R 2}-\bar{t}_{R 1} \bar{t}_{L 2}=0$. Then, making use of this relation in Eq. (17), one can extract the on-resonance transfer matrix

$$
\mathcal{T}(\varepsilon=\tilde{E}=0) \equiv\left(\begin{array}{cc}
0 & -\bar{t}_{L 1}^{*} / \bar{t}_{R 1}^{*} \\
\bar{t}_{R 2} / \bar{t}_{L 2} & 0
\end{array}\right)
$$

Using the relation between the transfer matrix $\mathcal{T}$ and the transmission, Eq. (7), one obtains

$$
T=\frac{2 i \bar{t}_{L 1}^{*} \bar{t}_{R 2}}{\bar{t}_{L 1}^{*} \bar{t}_{L 2}+\bar{t}_{R 2} \bar{t}_{R 1}^{*}} .
$$


If the tunneling elements differ only by a phase, $\bar{t}_{L 1}=\bar{t}_{L 2} \equiv \bar{t} e^{i \theta_{L}}$ and $\bar{t}_{R 1}=\bar{t}_{R 2} \equiv \bar{t} e^{i \theta_{R}}$, with $\theta_{R}, \theta_{L}, \bar{t} \in \Re$, then the only possible solution is $|T|^{2}=1$ (full transmission). This is in general the case of a double arm interferometer.

Now, we will consider the second case, $A \neq 0$. Notice that this is typically the situation in Eq. (11). The only possible solution of the system of Eqs. (17) is then $C_{0}=C_{1}=0$, i.e., from the boundary conditions (6) follows that $R=-1$ and $T=0$ (full reflection). If we assume a phase difference between the tunneling elements, i.e., $\bar{t}_{L 1}=\bar{t}_{L 2} \equiv \bar{t}$ and $\bar{t}_{R 1}=\bar{t} e^{i \theta_{1}} ; \quad \bar{t}_{R 2}=\bar{t} e^{i \theta_{2}}$ with $\theta_{i}, \bar{t} \in \Re$, the amplitudes on the orbital levels 1 and 2 are given by

$$
\begin{gathered}
\bar{D}_{1}=\frac{-2 i e^{i \theta_{2}}}{\bar{t}\left[1-e^{i\left(\theta_{1}-\theta_{2}\right)}\right]} \\
\bar{D}_{2}=\frac{2 i e^{i\left(\theta_{1}-\theta_{2}\right)}}{\bar{t}\left[1-e^{i\left(\theta_{1}-\theta_{2}\right)}\right]} .
\end{gathered}
$$

Notice that Eq. (21) implies that the probability of finding the electron on each of the degenerate levels is the same

It is worth mentioning that the dips in the conductance are inherent to the two-channel resonant tunneling, $\underline{\underline{33}}$ and they have been described in double-dot Aharonov-Bohm interferometers even at finite temperatures and in the presence of electron-electron interactions. $\underline{34}, \underline{35}$

\section{CURRENT AND CONDUCTANCE}

To study the current through the system formed by the triple dot and the two leads we apply the Landauer-Büttiker formula $\cdot \underline{36}, \underline{37}, 38$ If the chemical potential of the left lead is $\mu_{L}$ and a bias voltage $\Delta V / e=\left(\mu_{L}-\mu_{R}\right) / e$ is applied between the two leads, the current flowing through the system at zero temperature is given by

$$
I(\Delta V, \phi)=\frac{e}{h} \int_{\mu_{L}-\Delta V}^{\mu_{L}} d \varepsilon|T(\varepsilon, \Delta V, \phi)|^{2},
$$

while the differential conductance can be obtained as

$$
G=\frac{\partial I(\Delta V, \phi)}{\partial \Delta V / e}=\frac{G_{0}}{2}\left[\left|T\left(\varepsilon=\mu_{L}-\Delta V, \Delta V, \phi\right)\right|^{2}+\int_{\mu_{L}-\Delta V}^{\mu_{L}} d \varepsilon \frac{\partial}{\partial \Delta V}|T(\varepsilon, \Delta V, \phi)|^{2}\right] .
$$

It relates the zero-temperature conductance to the transmission probability $|T(\varepsilon, \Delta V, \phi)|^{2}$ at incident energy $\varepsilon$. Here $G_{0}=\frac{2 e^{2}}{h}$ is the quantum of conductance. Notice that in the linear 
regime (small $\Delta V$ ), the differential conductance (or just conductance) is proportional to the transmission at the Fermi level of the left lead, since the second term in Eq. (23) cancels for $\Delta V \rightarrow 0$, while at intermediate bias, the second term is responsible of extra structure in the peaks of the linear conductance, increasing the complexity of the profile as we increase the bias. $\frac{39}{}$ The current and the conductance in the linear regime are given by

$$
\begin{gathered}
I=\frac{e}{h}\left|T\left(\varepsilon=\mu_{L} ; 0, \phi\right)\right|^{2} \Delta V \\
G=\frac{G_{0}}{2}\left|T\left(\varepsilon=\mu_{L} ; 0, \phi\right)\right|^{2} .
\end{gathered}
$$

Therefore, the problem of obtaining the current through the system is reduced to the calculation of the transmission coefficient $T(\varepsilon ; \Delta V, \phi)$.

\section{RESULTS}

Here we are interested in the regime in which the energy band of the leads is much bigger than the energy splitting between the three levels of the TQD $(|t|<<1)$. Also, the tunneling between the dots and the leads will be taken much smaller than other energy scales involved in the problem.

Let us consider first the linear transport, where Eqs. (25) and (24) are valid. Fig. 5(a) shows the transmission probability at the Fermi energy versus the magnetic flux and the dot energy $E$ for tunneling $t_{L 2}=0$ with $t=-0.2, t_{L 1}=t_{R 3}=t_{L D}=-0.05$, and $E_{F}=-1$. As shown in Fig. 5(a), the transmission is periodic in the magnetic flux with period of one flux quantum. The transmission pattern can be understood as follows. Electrons tunnel through the TQD only when one of the three levels of the quantum molecule is on-resonance with the Fermi energy $\left(E_{F}=-1\right)$. For an arbitrary value of the magnetic flux, this occurs for three different values of the dot energy $E$. For example, at zero magnetic flux the resonance condition is fulfilled when the ground state $\left(E_{G}=E-2|t|\right)$ is on-resonance with the Fermi level $(E=-0.6)$ while in the case of the doubly-degenerated excited state, $\left(E_{e}=E+|t|\right)$, this happens when $E=-1.2$. The oscillation in the levels of the isolated triple dot with the magnetic flux is reflected in the transmission since the values of high transmission correspond to dot energies on-resonance with the Fermi level. The structure that appears in Fig. 5(a) is preserved for values of $\left|t_{L D}\right| \leq|t|$, with the width of the high transmission regions increasing with $t_{L D}$. For $\left|t_{L D}\right|>|t|$, the transmission is a smoother function of the flux (not shown 
here) and the profile is deformed with respect to the case considered here. Fig. 5(b) shows the transmission as a function of the magnetic flux and dot energy when the tunneling between the left lead and the second dot is allowed $\left(t_{L 2}=t_{L D}\right)$ for the ratio $\phi^{\prime} / \phi=1.73$. This ratio of fluxes leads to a non-periodic structure superimposed on the one appearing in Fig. 5(a), a consequence of the interference between the two magnetic fluxes.

Let us consider now a case in which the transmission window given by $\Delta V$ is much bigger than $|t|$ (this is the case in most experimental setups with networks of lateral dots, including Ref. 3). Then, the total current contains contributions from all incident energies within the transmission window, see Eqs. (22) and(23), and corresponds to the non-linear regime. Fig. 66 shows a contour plot of the differential conductance versus the magnetic flux and the dot energy for $E_{F}=-1$ and $\Delta V=1$. The case depicted corresponds to $t_{L 2}=t_{L D}=-0.05$. The variation of $G$ with the flux $\phi$ resembles the dependence of the transmission probability, shown in Fig. [5(b), allowing the determination of the tunneling matrix elements $|t|$ from the amplitude of the oscillations. Therefore, the differential conductance under finite sourcedrain bias maps out the energy levels of the TQD.

Although the contour plot of Fig. 66 provides the basic picture of the behaviour of the TQD connected to the leads, it does not allow us to see several important details. To simplify the analysis of the fine structure we can consider the simplest case with single lead-to-dot connection and look at the current. We have depicted the resulting current for three different values of the dot energy, $E=-0.8$, Fig. 7 (a), $E=-0.6$, Fig. 7(b), and $E=-0.2$, Fig. 7(c), using the same parameters as in Fig. 6. The first case, $E=-0.8$, corresponds to the scenario where the three levels of the triple dot can contribute to the current. As we have shown previously, when the incident particle has an energy on-resonance with degenerate levels, the transmission probability drops to zero. In fact, even for the general case where $t_{L 2} \neq 0$ and under an applied bias $\Delta V$, it can be proven that close to the central energy $\tilde{E}$

$$
\left|T\left(\varepsilon, \Delta V, \phi=\frac{n}{2}\right)\right|^{2} \approx f(E, \Delta V)(\varepsilon-\tilde{E})^{2}, \quad \phi=\frac{n}{2}, n=1,2 \ldots
$$

where $f(E, \Delta V)$ is a function of the dot energy and the bias voltage. These zeros in the transmission are reflected in the current as sharp drops whenever a pair of degenerate levels lies between the chemical potential of the two leads. In Fig. 7(a), this happens when $\phi=n / 2, n=1,2, \ldots$. It should be pointed out that the zero in the transmission probability at the degenerate level does not imply zero current, since the large applied bias leads to 
a contribution from all three levels. Anomalous behaviour in the transmission through a double-dot Aharonov-Bohm interferometer have also been described in Ref. 34,40. In particular, Kubo et al. $\stackrel{40}{\underline{6}}$ have reported sharp zero conductance dips in the linear regime. A second scenario appears in Fig. 7(b), where only two levels can contribute to the current. In this case, the anomalous dips in the current appear with a periodicity of one flux quantum. Finally, the third possibility, at most one level contributing to the current, is presented in Fig. 7(c). Here, the dips in the current have disappeared since the possible degenerate states of the triple dot are outside the transmission window.

From our discussion, it should be clear that the anomalous behaviour of the current with the magnetic field is a manifestation of degeneracies in the system or, in other words, that the presence of strong dependencies of the conductance with the magnetic field is indicative of degeneracies in the system.

\section{CONCLUSIONS}

Summarizing, we have analyzed the linear and non-linear differential conductance through an equilateral triple dot connected to two leads and subject to a perpendicular magnetic field. Two possible spatial configurations were analyzed: a single lead-to-dot connection where only one flux threads the system and a double connection where two different fluxes must be considered. In both cases, we found that superimposed on the AB oscillations induced by resonances with the oscillatory levels of the TQD, sharp dips in the current appear whenever degenerate states lie between the chemical potential of the two leads. Therefore, three scenarios are possible: no dips (degeneracies outside the transmission window), dips appearing with a periodicity of one flux quanta (at most two level contributing to the current) and dips with periodicity of half flux quantum (all three levels contributing). We provided a simple theory of the dips in the conductance. The presence of a double lead-to-dot connection produces an additional non-periodic structure in the conductance as a function of the magnetic field, related to the existence of two non-commensurate fluxes threading the system. Both effects, AB oscillations and the dips in the current are also apparent when large potential bias is applied between the two leads. 


\section{Acknowledgments}

The Authors acknowledge support by the Canadian Institute for Advanced Research and QuantumWorks, and also useful discussions with A. Sachrajda, L. Gaudreau, S. Studenikin, Y.-P. Shim and M. M. Korkusisnki.

1 L. Gaudreau, S. Studenikin, A. S. Sachrajda, P. Zawadzki, A. Kam, J. Lapointe, M. Korkusinski, and P. Hawrylak, Phys. Rev. Lett. 97, 036807 (2006).

2 M. Korkusinski, I. Puerto Gimenez, P. Hawrylak, L. Gaudreau, S. A. Studenikin, and A. S. Sachrajda, Phys. Rev. B 75, 115301 (2007).

3 L. Gaudreau, A. S. Sachrajda, S. Studenikin, P. Zawadzki, A. Kam, and J. Lapointe, ICPS Conf. Proc., to be published (2007).

4 T. Ihn, M. Sigrist, K. Ensslin, W. Wegscheider, and M. Reinwald, New J. Phys. 9, 111 (2007).

5 D. Goldhaber-Gordon, J. Göres, M. A. Kastner, H. Shtrikman, D. Mahalu, and U. Meirav, Phys. Rev. Lett. 81, 5225 (1998).

6 S. M. Cronenwett, T. H. Oosterkamp, and L. P. Kouwenhoven, Science 281, 540 (1998).

7 J. Schmid, J. Weis, K. Eberl, and K. von Klitzing, Physica (Amsterdam) 256B-258B, 182 (1998).

8 A. C. Hewson, The Kondo Problem to Heavy Fermions (Cambridge University Press, Cambridge, UK, 1993).

9 S. Hershfield, J. H. Davies, and J. W. Wilkins, Phys. Rev. Lett. 67, 3720 (1991).

10 Y. Meir and N. S. Wingreen, Phys. Rev. Lett. 68, 2512 (1992).

11 A. P. Jauho, N. S. Wingreen, and Y. Meir, Phys. Rev. B. 50, 5528 (1994).

12 A. L. Yeyati, A. Martín-Rodero, and F. Flores, Phys. Rev. Lett. 71, 2991 (1993).

13 M. H. Hettler and H. Schoeller, Phys. Rev. Lett. 74, 4907 (1995).

14 R. Peierls, Z. Phys. 80, 763 (1933).

15 J. M. Luttinger, Phys. Rev. 84, 814 (1951).

16 Y. Gefen, Y. Imry, and M. Y. Azbel, Phys. Rev. Lett 52, 129 (1984).

17 M. Büttiker, Y. Imry, and M. Y. Azbel, Phys. Rev. A 30, 1982 (1984).

18 R. G. Chambers, Phys. Rev. Lett. 5, 3 (1960). 
19 A. Tonomura, T. Matsuda, R. Suzuki, A. Fukuhara, N. Osakabe, H. Umezaki, J. Endo, K. Shinagawa, Y. Sugita, and H. Fujiwara, Phys. Rev. Lett. 48, 1443 (1982).

20 R. A. Webb, S. Washburn, C. P. Umbach, and R. B. Laibowitz, Phys. Rev. Lett. 54, 2696 (1985).

21 A. Yacoby, M. Heiblum, V. Umansky, H. Shtrikman, and D. Mahalu, Phys. Rev. Lett. 73, 3149 (1994).

22 Y. Ji, Y. Chung, D. Sprinzak, M. Heiblum, D. Mahalu, and H. Shtrikman, Nature 422, 415 (2003).

23 B. L. Altshuler, A. G. Arnov, and B. Z. Spivak, JETP Lett. 33, 94 (1981).

24 V. Chandrasekhar, M. J. Rooks, S. Wind, and D. E. Prober, Phys. Rev. Lett. 55, 1610 (1985).

25 K. Ingersent, A. W. W. Ludwig, and I. Affleck, Phys. Rev. Lett. 95, 257204 (2005).

26 T. Kuzmenko, K. Kikoin, and Y. Avishai, Phys. Rev. Lett. 96, 046601 (2006).

27 Z.-T. Jiang and Q.-F. Sun, J. Phys.: Condens. Matter 19, 156213 (2007).

28 C. W. Groth, B. Michaelis, and C. W. J. Beenakker, Phys. Rev. B 74, 125315 (2006).

29 B. Michaelis, C. Emary, and C. W. J. Beenakker, Europhys. Lett. 73, 677 (2006).

30 C. Emary, cond-mat/0705.2934.

31 G. D. Mahan, Many-Particle Physics (Plenum Press, New York, 1990).

32 U. Fano, Phys. Rev. 124, 1866 (1961).

33 T. V. Shahbazyan and M. E. Raikh, Phys. Rev. B 49, 17123 (1994).

34 B. Kubala and J. König, Phys. Rev. B 65, 245301 (2002).

35 Y. Tokura, H. Nakano, and T. Kubo, New J. Phys. 9, 113 (2007).

36 R. Landauer, IBM J. Res. Dev. 1, 223 (1957).

37 R. Landauer, Philos. Mag. 21, 863 (1970).

38 M. Büttiker, Phys. Rev. Lett. 57, 1761 (1986).

39 E. Castaño, G. Kirczenow, and S. E. Ulloa, Phys. Rev. B. 42, 3753 (1990).

40 T. Kubo, Y. Tokura, T. Hatano, and S. Tarucha, Phys. Rev. B 74, 205310 (2006). 


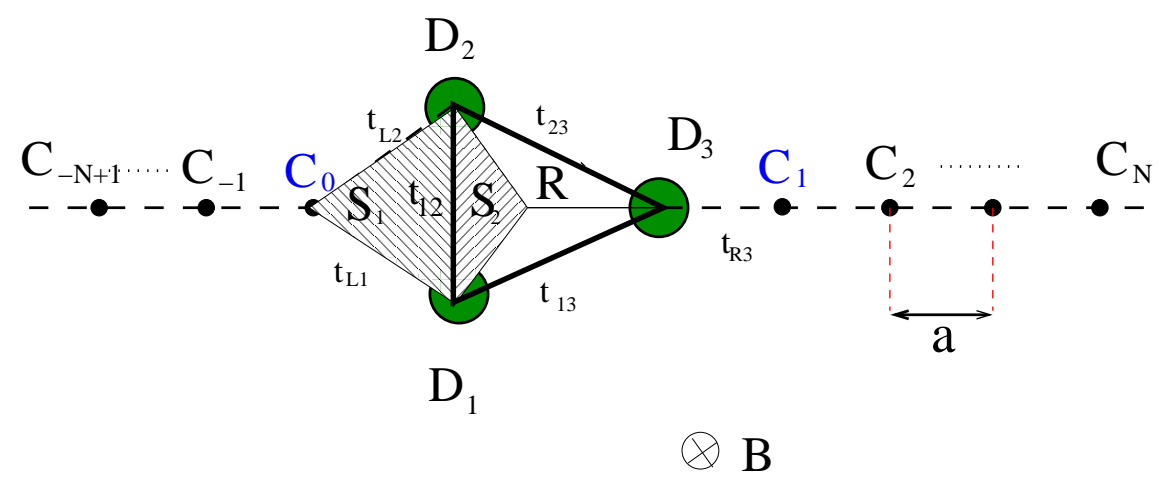

FIG. 1: Schematic diagram of the spatial layout of the triple dot and the two leads. Allowed tunneling between different sites is marked with thick long-dashed lines.

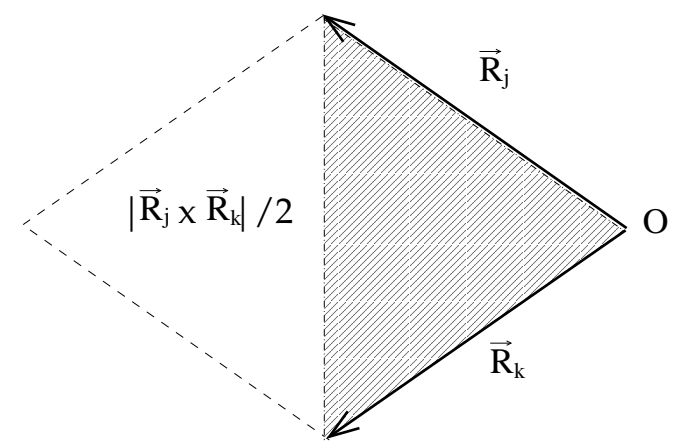

FIG. 2: Area responsible of the phase difference between two points $\mathbf{R}_{j}$ and $\mathbf{R}_{k}$ when a vector potential $\mathbf{A}$ with a gauge centered in the point $O$ is considered. 


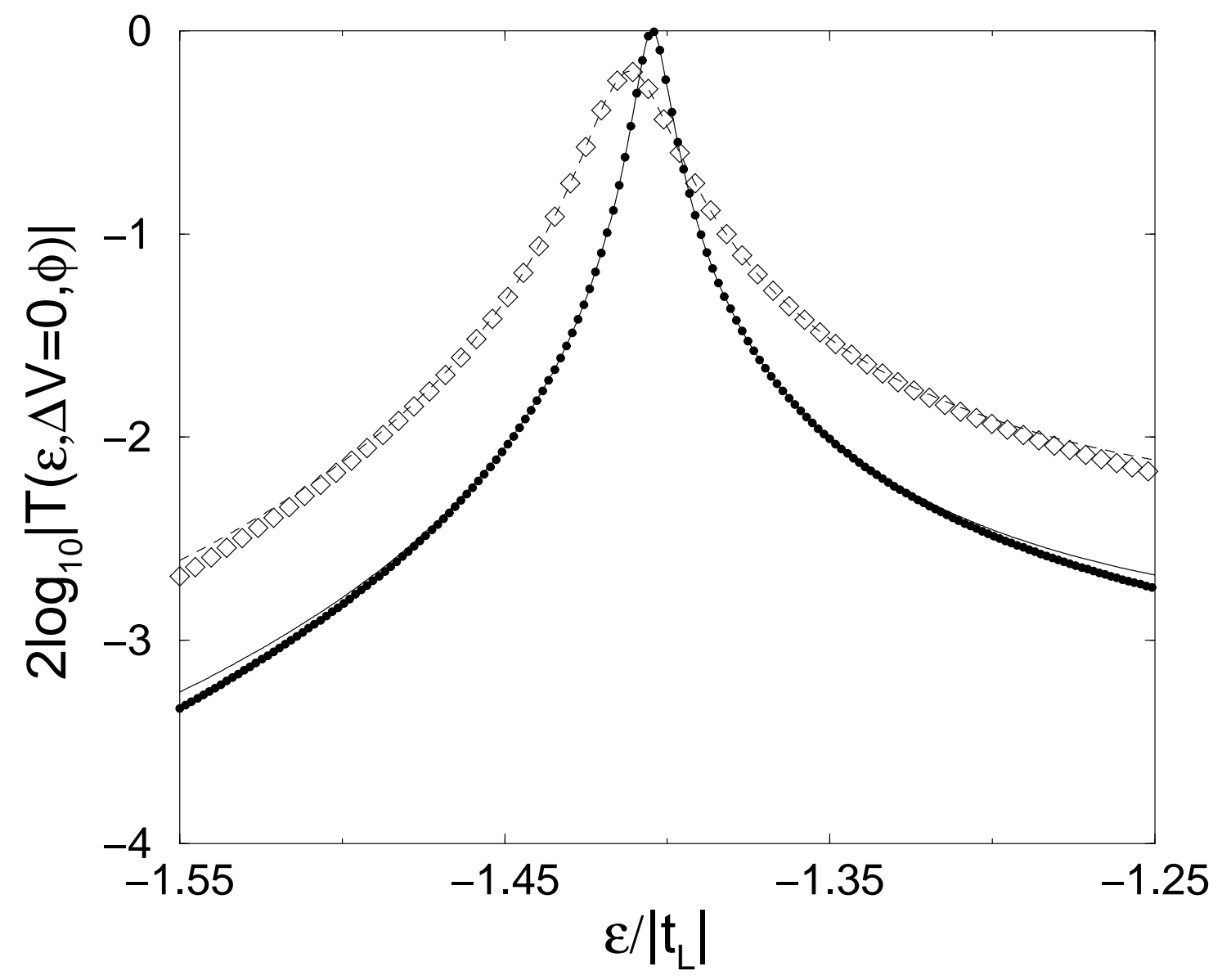

FIG. 3: $\log _{10}$ of the transmission probability at zero magnetic field versus the incident energy close to the on-resonance condition with the ground state of the triple dot. Solid line corresponds to single lead-to-dot connection and dashed line indicates the double connection. Dots (diamonds) shows a fitting to a Fano line shape with $q=86.73$ and $\Gamma=9.60 \times 10^{-3}\left(q=34.18\right.$ and $\left.\Gamma=2.40 \times 10^{-2}\right)$. The other parameters are $E=-1, \Delta V=0, t=-0.2, t_{L 1}=t_{R 3}=-0.05$ and $\phi^{\prime} / \phi=1.73$ for the double connection. 

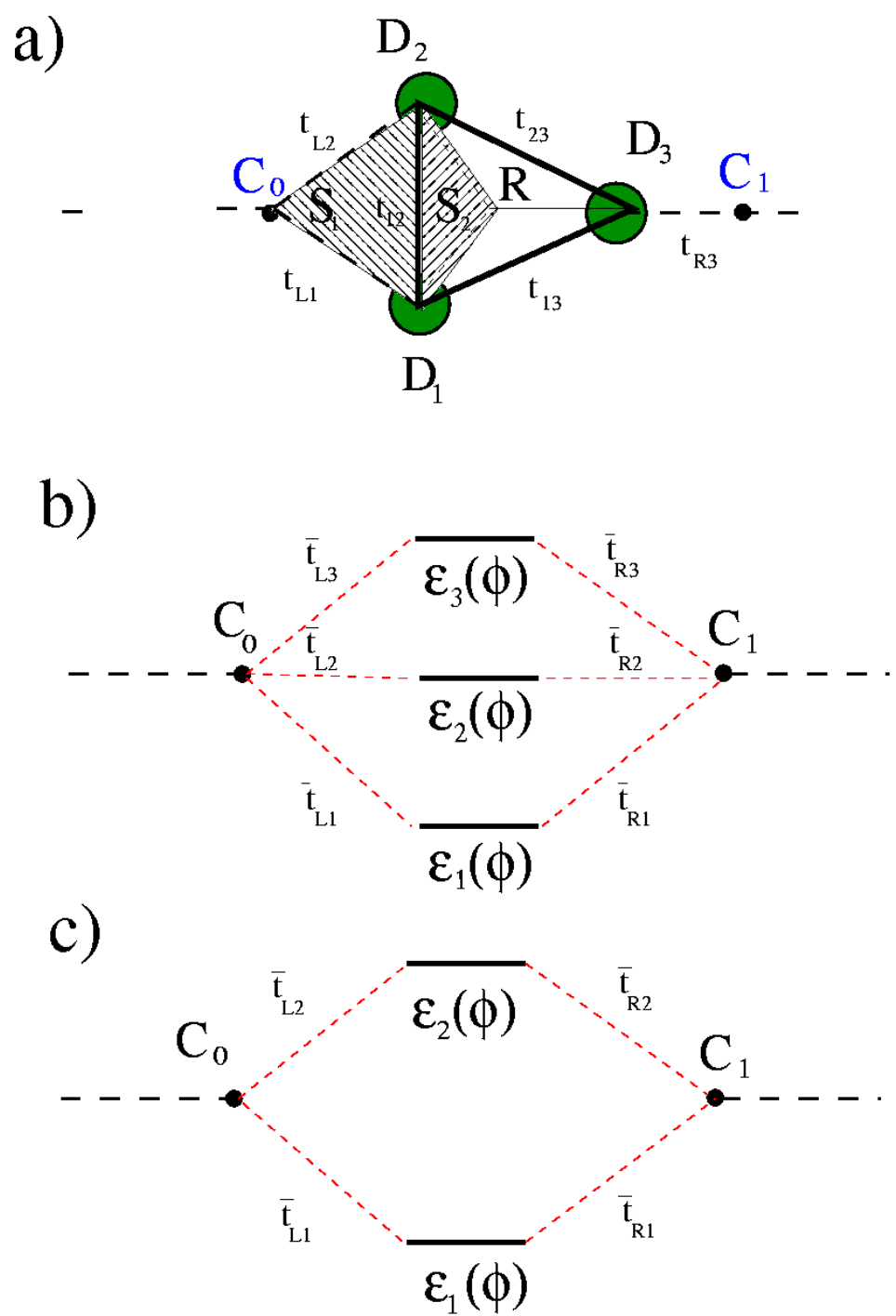

FIG. 4: Schematic representation of the amplitudes and hopping matrix elements between sites: a) in the original model, b) in the bases of eigenvectors of the isolated triple dot and $\mathbf{c}$ ), the simplified version that accounts for the case where the incident energy is close to a quasi-degenerate pair of levels levels, i.e., $\varepsilon \approx \epsilon_{1}(\phi) \approx \epsilon_{2}(\phi)$. 

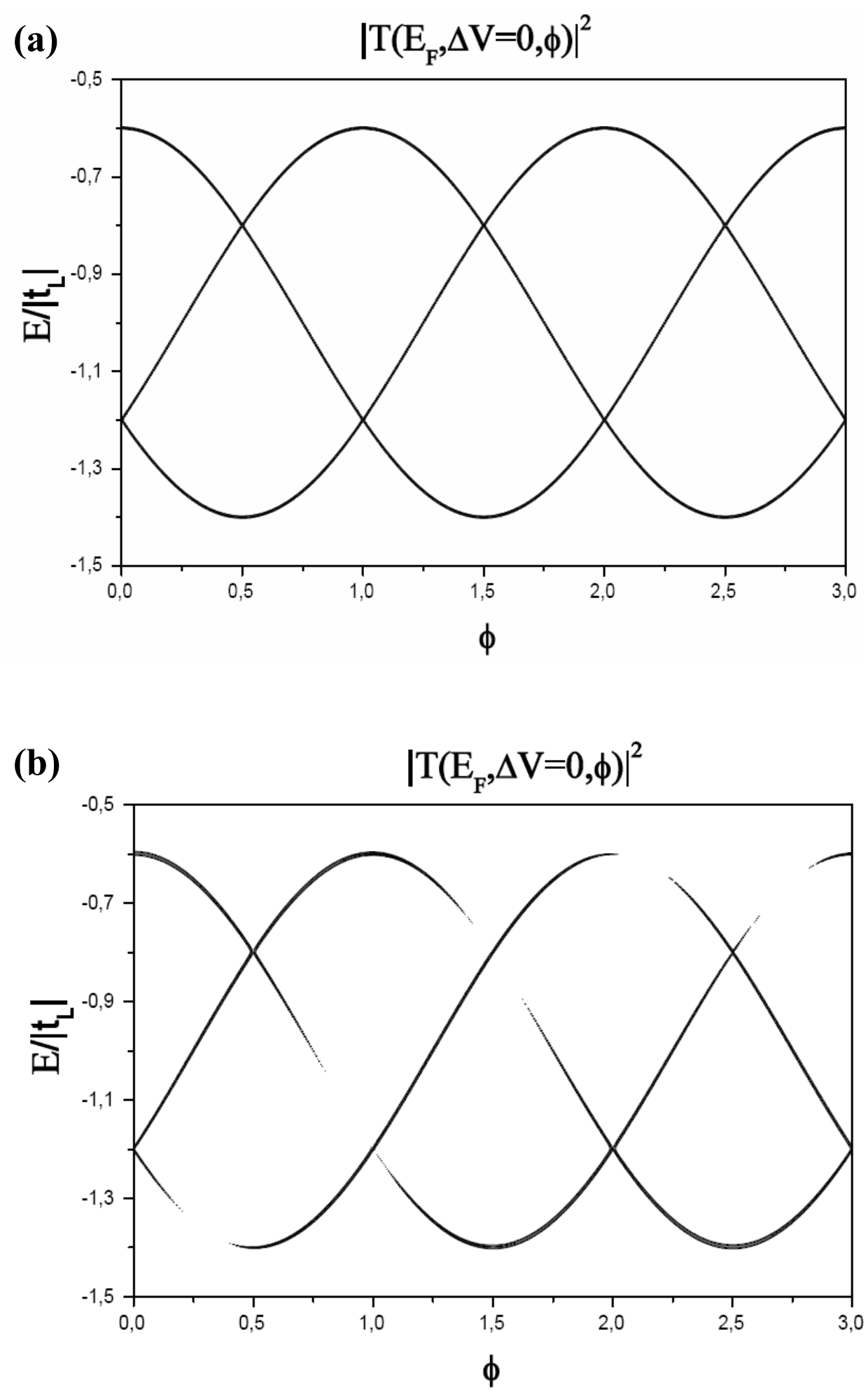

FIG. 5: Transmission probability (vertical gray scale with black for 1) at the Fermi energy versus the number of magnetic flux quanta $\phi$ and dot energy $E$ for the cases (a) $t_{L 2}=0$ showing periodic behaviour with $\phi$ and (b), $t_{L 2}=t_{L D}$ with additional non-periodic structure. $t_{L D}=-0.05, \Delta V=0$ and $E_{F}=-1$. 


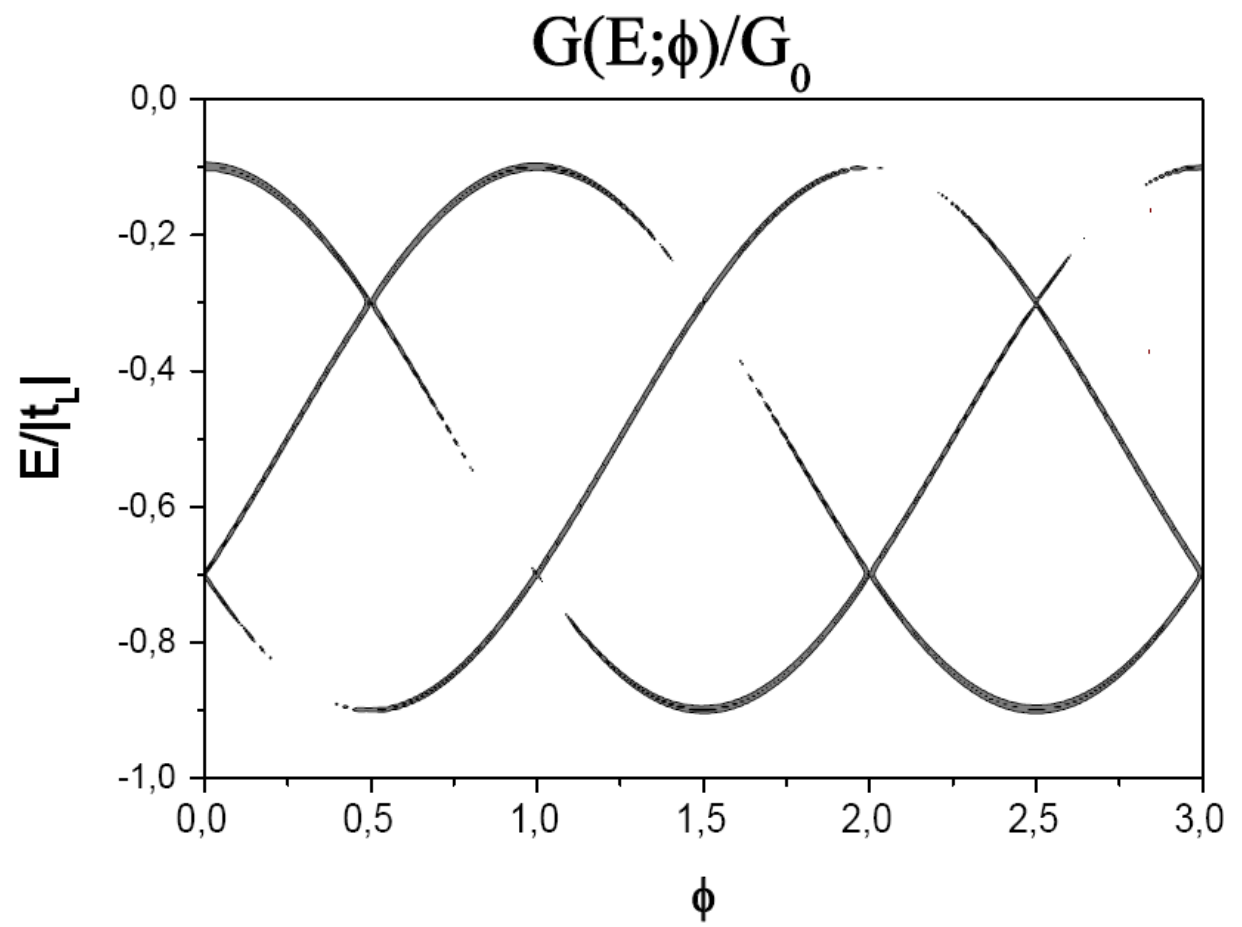

FIG. 6: Differential conductance $G$ (vertical gray scale) versus number of magnetic flux quanta $\phi$ and the dot energy $E$ in the non-linear regime. $\Delta V=1, \mu_{L}=-1, t=-0.2$ and $t_{L 2}=t_{L D}=-0.05$. The ratio of fluxes is $\phi^{\prime} / \phi=1.73$. 
(a)

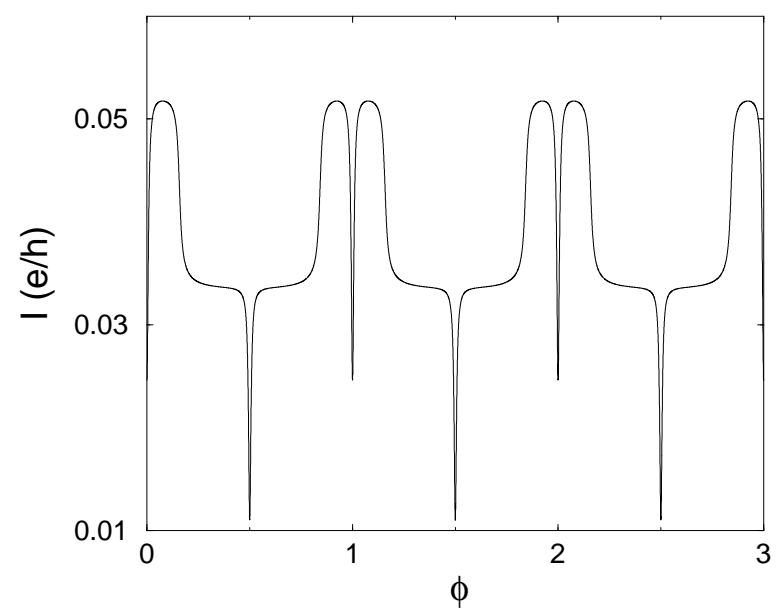

(b)

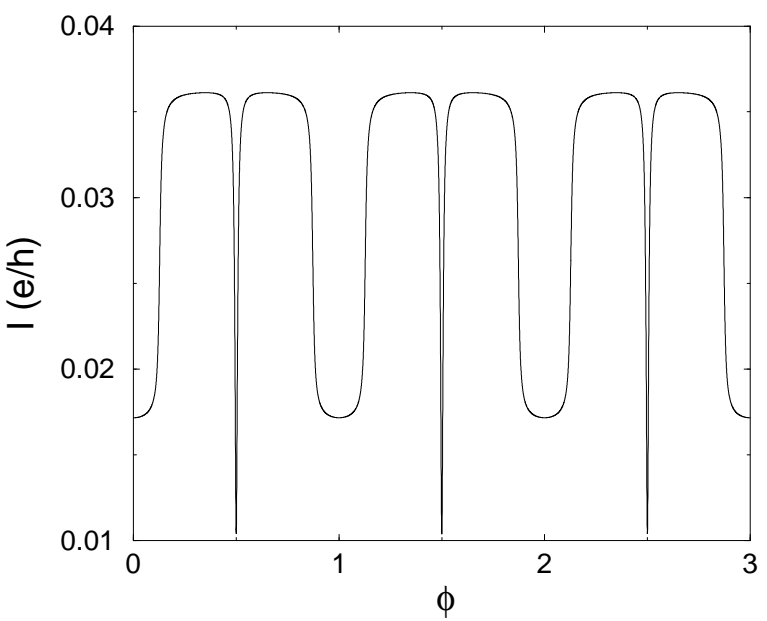

(c)

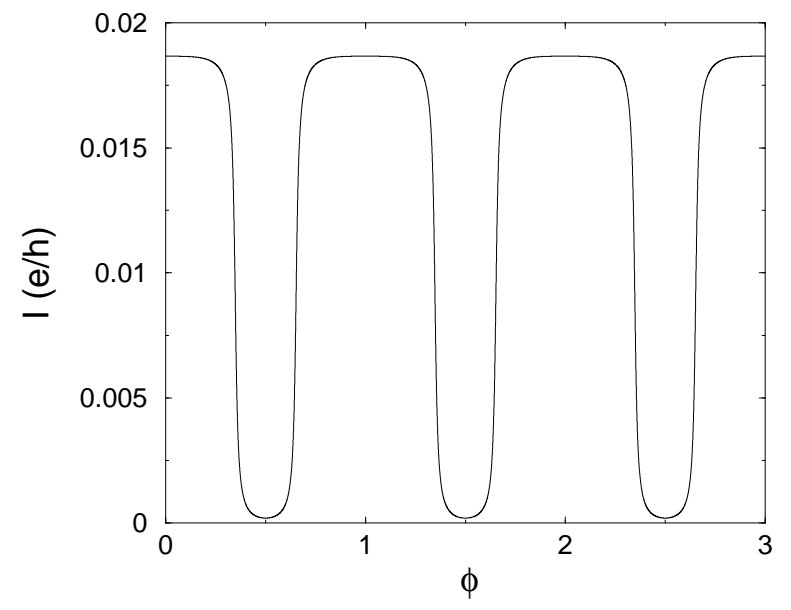

FIG. 7: Current versus number of magnetic flux quanta $\phi$ in the non-linear regime under a bias $\Delta V=1$ and for the single lead-to-dot connection. Fig. (a) shows the case where the three levels can contribute to the current, with $E=-0.8$, Fig. (b), up to two levels $(E=-0.6)$ and Fig. (c) only one level $(E=-0.2) . E_{F}=-1, t=-0.2$, and $t_{L D}=-0.05$. The periodicity of the drops in the current changes for each case: $\phi_{0} / 2$ in (a), $\phi_{0}$ in (b) and no drops in (c). 